\title{
Exploring Correlation between Extent of Tumour Necrosis on MRI And Histological Grade of Soft Tissue Sarcoma - An Initial Experience
}

\author{
S. Thaker ${ }^{1}$, C. Azzopardi², A. Gulia ${ }^{3}$, R. Botchu² \\ 1 Department of Diagnostic Imaging, Kettering General Hospital, Kettering UK \\ 2 Department of Musculoskeletal Radiology, Royal Orthopaedic Hospital, Birmingham, UK \\ 3 Department of Orthopaedic Oncology, Tata Memorial Centre, Mumbai, India
}

\section{CORRESPONDING AUTHOR:}

Rajesh Botchu

Department of Musculoskeletal Radiology

The Royal Orthopedic Hospital

Bristol Road South

B31 2AP Northfield

Birmingham, UK

E-mail: drbrajesh@yahoo.com

DOI:

10.32098/mltj.04.2020.11

LEVEL OF EVIDENCE: 4

\begin{abstract}
SUMMARY
Objective. Soft Tissue Sarcoma (STS) is a rare form of cancer which includes a variety of malignant connective tissue neoplasms with high morbidity and mortality. Amongst these, undifferentiated pleomorphic sarcoma - accounting for up to $20 \%$ of all sarcoma - lacks a specifically identified line of cellular differentiation. Magnetic Resonance imaging (MR) have been used for local staging of STS, determining tumour extent, tumour characteristics, and neurovascular bundle infiltration. In addition to histologic evaluation following percutaneous biopsy, MR can possibly be used as a non-invasive investigation to determine tumour grade as it has been successfully used as a surrogate marker for other malignant tumours, for example: brain tumours. We evaluated similar MRI utility for undifferentiated pleomorphic sarcoma to assess necrosis as a possible predictor of histological grading.

Methods. We evaluated 47 cases of histologically proven undifferentiated pleomorphic sarcoma. We hypothesized that there was a direct correlation between the degree of tumour necrosis on MR and histologic grade. Two experienced consultant musculoskeletal radiologists blinded to histological grade of tumour evaluated MR images retrospectively. The extent of tumour necrosis was categorised as $<25 \%-$ MR grade I, $26-50 \%$ - MR grade II, $51-75 \%$ - MR grade III and $>76 \%$ - MR grade IV. Final pathologic grading using Fédération Nationale des Centres de Lutte le Cancer (FNCLCC) classification were obtained for all patients which divide tumour grades into low (grade I) and high (grade II and III) category and correlated with MRI results.

Results. 17 (36.1\%) patients with MR Necrosis grade I, 8 (17.02\%) patients with MR necrosis grade II and $10(21.27 \%)$ patients with MR necrosis grade III, were highgrade STS (grade III significantly higher than grade II) histologically. Whereas, out of $12(25.53 \%)$ patients with MR necrosis grade IV demonstrating more than $75 \%$ tumour necrosis, $1(2.12 \%)$ patient was low-grade (FNCLCC grade I) and rest were high-grade STS including $1(2.12 \%)$ patient of grade II and $10(21.27 \%)$ patients of grade III.

Conclusions. We were unable to establish positive or negative correlation between the degree of necrosis on MR and histological grade of STS on this cohort study.
\end{abstract}

\section{KEY WORDS}

Soft tissue sarcoma; necrosis; grade; undifferentiated pleomorphic sarcoma; histological grade; tumour necrosis; MRI grading. 


\section{INTRODUCTION}

Soft Tissue Sarcoma (STS) is a rare form of cancer which includes a variety of malignant connective tissue neoplasms with high morbidity and mortality. In the UK, in 2010, 3272, new cases of STS were diagnosed. Once diagnosed with these inherently aggressive neoplasms, the survival rate in STS patients steadily declines with time showing 10 years age-standardised relative survival rate for STS is $45 \%$ in the UK $(1,2)$.

Soft tissue sarcoma are further classified into numerous categories depending upon predominant tissue. These include leiomyosarcoma, liposarcoma, fibroblastic sarcoma including undifferentiated pleomorphic sarcoma (previously known as Malignant Fibrous Histiocytoma (MFH)), rhabdomyosarcoma, soft tissue Ewing's sarcoma, synovial sarcoma, vascular sarcoma, malignant peripheral nerve sheath tumours and other rarer forms of sarcoma which are not otherwise specified (sarcoma NOS) (1).

MRI is usually the imaging investigation of choice for sarcoma, determining tumour extent, tumour characteristics (figure 1) and neurovascular bundle infiltration, characterising lesions and prognosticating them. Tumour necrosis has been successfully used as a surrogate marker for different malignant tumours, for example, brain tumours. Our effort was to evaluate similar MRI utility for undifferentiated pleomorphic sarcoma to assess necrosis as a possible predictor of pathological grading.

\section{MATERIALS AND METHODS}

After institutional board review approval, we created a retrospective search of prospectively maintained radiology database at our institution and found 47 cases of histologically proven undifferentiated pleomorphic sarcoma (3). We hypothesised that there was a correct direct relation between the degree of tumour necrosis on MR and histologic grade. Two experienced consultant musculoskeletal radiologists who were blinded to histological grade of tumour evaluated MR images retrospectively. The extent of tumour necrosis was categorised as $<25 \%-$ MR grade I, $26-50 \%-$ MR grade II, $51-75 \%$ - MR grade III and $>75 \%$ - MR grade IV. Final pathologic grading using Fédération Nationale des Centres de Lutte le Cancer (FNCLCC) classification (4) modified initially from Trojani classification, were obtained for all patients which divide tumour grades into low (grade I) and high (grade II and III) category.

\section{RESULTS}

We have compiled a dataset for 47 patients comparing necrosis on MR imaging with histological grading (table I).

Amongst 17 (36.1\%) patients with MR Necrosis grade I, $8(17.02 \%)$ patients with MR necrosis grade II and 10 $(21.27 \%)$ patients with MR necrosis grade III, all turned out to be high-grade STS (grade III significantly higher than grade II) on histologic grading. Whereas, in 12

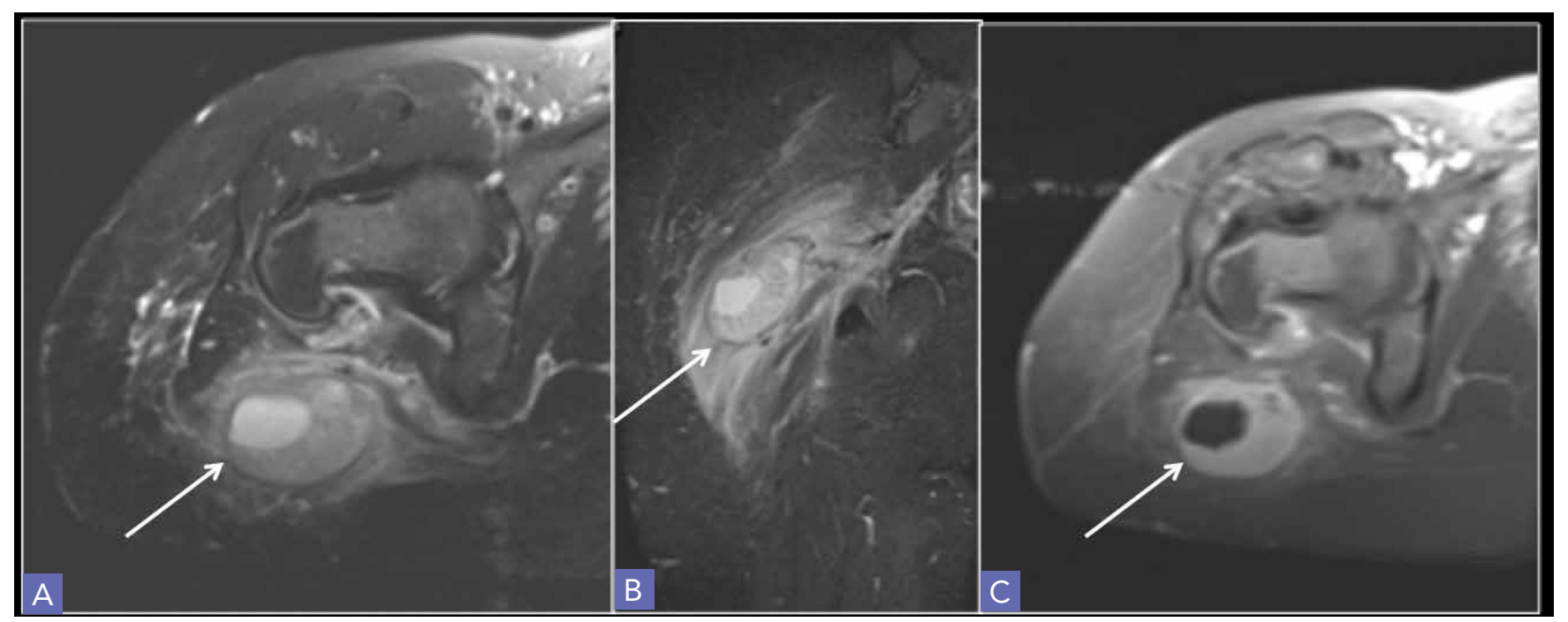

Figure 1. STIR axial (a) and coronal (b) and T1 fat suppressed post contrast axial (c) images of the right hip demonstrates necrotic tumour with MR necrosis grade 1 which was histologically grade III. 
Table I. Demonstrates the grade of tumour and degree of necrosis. (\# Tumour necrosis on MR imaging evaluated by two blinded musculoskeletal radiologists, percentage of necrosis is calculated against whole-tumour volume in a semi-quantitative manner; * Histologic grading using Fédération Nationale des Centres de Lutte le Cancer (FNCLCC) classification).

\begin{tabular}{|l|l|l|l|l|l|}
\hline $\begin{array}{l}\text { MR } \\
\text { necrosis } \\
\text { grade\# }\end{array}$ & $\begin{array}{l}\text { Histologic } \\
\text { grade* }\end{array}$ & Grade I (Low-grade) & Grade II (High-grade) & Grade III (High-grade) & Total \\
\hline $\begin{array}{l}\text { MR grade I (Necrosis } \\
\text { <25\%) }\end{array}$ & $0(0.00 \%)$ & $3(6.38 \%)$ & $14(29.78 \%)$ & $17(36.1 \%)$ \\
\hline $\begin{array}{l}\text { MR grade II (Necrosis } \\
26-50 \%)\end{array}$ & $0(0.00 \%)$ & $2(4.25 \%)$ & $6(12.76 \%)$ & $8(17.02 \%)$ \\
\hline $\begin{array}{l}\text { MR grade III (Necrosis } \\
51-75 \%)\end{array}$ & $0(0.00 \%)$ & $3(6.38 \%)$ & $7(14.89 \%)$ & $10(21.27 \%)$ \\
\hline $\begin{array}{l}\text { MR grade IV (Necrosis } \\
>75 \%)\end{array}$ & $1(2.12 \%)$ & $1(2.12 \%)$ & $10(21.27 \%)$ & $12(25.53 \%)$ \\
\hline Total & $1(2.12 \%)$ & $9(19.15 \%)$ & $37(78.72 \%)$ & $47(100 \%)$ \\
\hline
\end{tabular}

$(25.53 \%)$ patients with MR necrosis grade IV demonstrating more than $75 \%$ tumour necrosis, $1(2.12 \%)$ patient was low-grade (FNCLCC grade I) and rest were highgrade STS including $1(2.12 \%)$ patient of grade II and 10 $(21.27 \%)$ patients of grade III (figure 1-3). We concluded that percentage of MR necrosis does not follow histological grading and in turn aggressiveness of the soft tissue sarcoma and we were unable to find correlation between percentage of MR tumour necrosis and histological grading, whether positive or negative.

\section{DISCUSSION}

STS tumours are inherently heterogeneous consisting of multiple tissue components that include cellular compartments, fat, cystic changes, and necrosis. MRI is an imaging investigation of choice for prognostication of STS tumours whereas histological tumour grading remains the most widely accepted prognostic biomarker, as it serves as the proxy marker of the relative risk of tumour metastasis and survival. Additionally, the histological grade of the tumour is used as a key factor to decide whether the

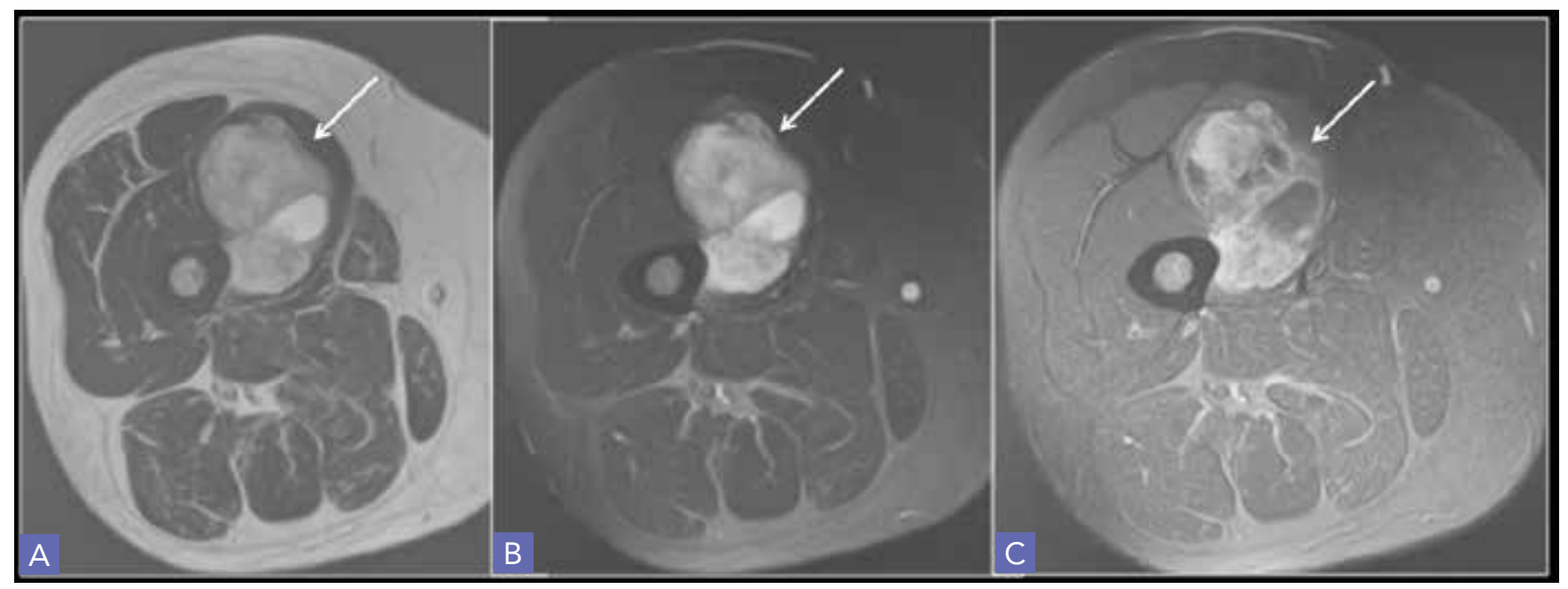

Figure 2. (a) T2, (b) STIR and (c) post-contrast T1 fat-suppressed axial images demonstrating irregular medial thigh compartment intramuscular neoplastic lesion showing less than $25 \%$ of the tumour necrosis in keeping with MR necrosis grade I. Histologically, the lesion was grade III demonstrating no direct positive correlation between MR necrosis and histological grading. 


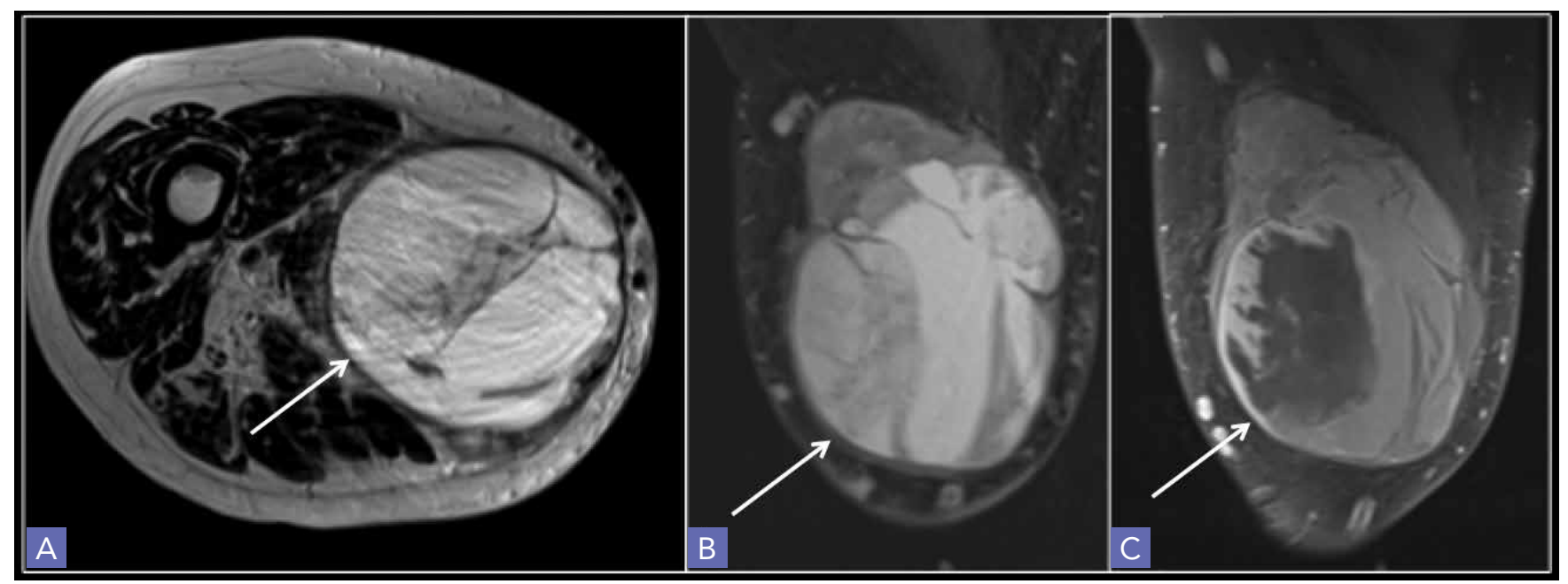

Figure 3. (a) T2 axial, (b) STIR coronal and (c) post contrast T1 fat-suppressed coronal images demonstrating irregular medial thigh compartment intramuscular neoplastic lesion showing approximately $75 \%$ of the tumour necrosis in keeping with MR necrosis grade III. Histologically, the lesion was grade III.

patient will need neoadjuvant chemotherapy in addition to surgery $(5,6)$.

Amongst these, undifferentiated pleomorphic sarcoma accounting for up to $20 \%$ of all sarcoma - is a particular category of STS, which lacks a specifically identified line of cellular differentiation when analysed by histological examination and immunohistochemical staining. Analysis of 70 cases of undifferentiated pleomorphic sarcoma showed highly complex with no specific recurrent aberration, making them impossible to categorise into more known sarcoma even at a genetic level (7). Similarly, it is not possible to characterise undifferentiated pleomorphic sarcoma solely on imaging as they do not demonstrate pathognomonic imaging characteristics.

Magnetic Resonance (MR) imaging, owing to its excellent contrast resolution, is the cornerstone imaging method for local staging (8). Several papers are looking at various MR imaging features and histological grading of the STS, but none is specifically looking at the extent of necrosis (9). According to Zhao et al., high-grade STS differs significantly from low-grade STS in terms of tumour size, tumour margin, heterogeneous signal intensity on T2-weighted images, and peritumoural high signal intensity. Presence of peritumoural contrast enhancement may act as a sole predictor of high-grade STS. Overall, in addition to histologic evaluation following percutaneous biopsy, MRI can be used as a supportive non-invasive investigation to determine tumour grade (10).

For brain tumours, tumour necrosis on MRI is poor prognostic factor and demonstrates direct positive correlation with histological grading and aggressiveness of the tumour. We used to exploit possibility of similar correlation for undifferentiated pleomorphic sarcoma. Contrary to the brain tumours, STS with less than $25 \%$ tumour necrosis could be high-grade histologically (figure 2) whereas it is also possible for STS with more than $75 \%$ tumour necrosis to be histologically low-grade or high-grade (figure 3). Hence, we were unable to establish correlation between MRI and histology, whether positive or negative.

We have searched multiple databases for similar studies and our study is, by far, the largest study evaluating correlation between MR necrosis and histological grading for proven cases of undifferentiated pleomorphic sarcoma. Possible limitations of this study may include small sample size, single centre experience and including only undifferentiated pleomorphic sarcoma. It is also possible that soft tissue sarcomas demonstrate completely different cellular lineage than brain tumours which may alter its growth characteristics including neoangiogenesis and their effect on tumour necrosis. However, we recommend multicentric study with a large sample size and involvement of more histological subtypes of soft tissue sarcomas to provide more robust statistical analysis.

\section{CONCLUSIONS}

In conclusion, we could not establish positive or negative correlation between the degree of necrosis on MR and histological grade of STS. Limitations of study were single centre pilot study, inclusion of only undifferentiated pleomorphic 
sarcoma and small sample size. We recommend further multicentric study with a larger sample size and other variety of soft tissue sarcoma.

\section{CONFLICT OF INTERESTS}

The authors declare that they have no conflict of interests.

\section{REFERENCES}

1. Cancer Research UK. Soft tissue sarcoma statistics. https:// www.cancerresearchuk.org/about-cancer/soft-tissue-sarcoma.

2. Iqbal A, McLoughlin E, Patel A, Davies AM, James SL, Botchu R. A diagnostic Approach to Deltoid Muscle Masses. MLTJ 2019;9(4):600-605.

3. Padulo J, Oliva F, Frizziero A, Maffulli N. Muscle, Ligaments and Tendons Journal - Basic principles and recommendations in clinical and field Science Research: 2018 update. MLTJ 2018;8(3):305-307.
4. Coindre JM. Grading of soft tissue sarcomas: review and update. Arch Pathol Lab Med 2006;130(10):1448-53.

5. Lucas DR, Kshirsagar MP, Biermann JS, et al. Histologic alterations from neoadjuvant chemotherapy in high-grade extremity soft tissue sarcoma: clinicopathological correlation. The oncologist 2008;13(4):451-8.

6. Menendez LR, Ahlmann ER, Savage K, Cluck M, Fedenko AN. Tumor necrosis has no prognostic value in neoadjuvant chemotherapy for soft tissue sarcoma. Clin Orthop Relat Res 2007;455:219-24.

7. J Jo VY, Fletcher CD. WHO classification of soft tissue tumours: an update based on the 2013 (4th) edition. Pathology 2014;46(2):95-104.

8. Walker EA, Salesky JS, Fenton ME, Murphey MD. Magnetic resonance imaging of malignant soft tissue neoplasms in the adult. Radiol Clin 2011;49(6):1219-34.

9. Liu QY, Li HG, Chen JY, Liang BL. Correlation of MRI features to histopathologic grade of soft tissue sarcoma. $\mathrm{Ai}$ Zheng 2008;27(8):856-60.

10. Zhao F, Ahlawat S, Farahani SJ, et al. Can MR imaging be used to predict tumor grade in soft-tissue sarcoma? Radiology 2014 8;272(1):192-201. 